\title{
DISTANCE AND VOLUME DECREASING THEOREMS FOR QUASICONFORMAL MAPPINGS
}

\author{
NICHOLAS C. PETRIDIS
}

\begin{abstract}
The method used by the author in deriving a Picard type theorem for quasiconformal mappings [Proc. Amer. Math. Soc. 61 (1976), 265-271], improved by a proposition of S.-T. Yau [Amer. J. Math. 100 (1978), 197-203] is employed here to extend the Schwarz-Ahlfors lemma to harmonic quasiconformal mappings. The target space is not necessarily hyperbolic, not even negatively curved.
\end{abstract}

1. Introduction. The following proposition, due to Ahlfors [7], gives an extension of the classical Schwarz lemma.

Proposition 1. Let $M$ be a one-dimensional Kaehler manifold with metric $d s_{M}^{2}$ whose Gaussian curvature is bounded above by a negative constant $-B$. Let $D_{a}$ be an open disc of radius a with metric given by

$$
d s_{D}^{2}=\frac{4 a^{2} d z d \bar{z}}{A\left(a^{2}-|z|^{2}\right)^{2}}
$$

(which makes $D_{a}$ a Kaehler manifold of constant curvature $-A<0$ ).

If $f: D_{a} \rightarrow M$ is a holomorphic mapping then

$$
f^{*} d s_{M}^{2}<\frac{A}{B} d s_{D}^{2}
$$

If $A<B$ the mapping is distance decreasing. In general we shall say that the mapping is distance (or volume) decreasing up to a constant.

Chern extended the lemma to holomorphic mappings between complex manifolds of higher dimension. The lemma was further extended, in the form of distance and volume decreasing theorems, by S. Kobayashi [7] and, more recently, by S. T. Yau [10]. Real analogues and generalizations of the lemma (as well as Liouville's and Picard's first theorem) have recently been obtained for harmonic mappings [2], quasiconformal mappings [5], and for mappings of bounded dilatation [4].

In all extensions of the Schwarz-Ahlfors lemma there is a variation of generality depending on the conditions imposed on the domain. The most general domain considered so far is that of Yau's [10], which is a complete Riemannian manifold with Ricci curvature bounded from below. The target space has been, almost invariably, assumed to be hyperbolic. In a recent paper [9] the author extended

Received by the editors December 10, 1979 and, in revised form, February 18, 1980.

AMS (MOS) subject classifications (1970). Primary 32H25, 53A99, 30A60.

Key words and phrases. Quasiconformal mappings, harmonic mappings, scalar curvature, SchwarzAhlfors lemma. 
Picard's first theorem to mappings with target space not necessarily hyperbolic. In the present work, taking advantage of a Yau's condition, the Schwarz-Ahlfors lemma is extended, in the form of distance and volume decreasing theorems, to mappings with target space not necessarily hyperbolic. The target space is even allowed to have some nonnegative sectional curvatures. The main results:

THEOREM 1. Let $M$ be an m-dimensional complete Riemannian manifold with Ricci curvature bounded below, and let $N$ be an n-dimensional Riemannian manifold with scalar curvature bounded from above by a fixed negative number $-C$. If the sectional curvatures of $N$ are bounded from above by a fixed number smaller than $C / m(m-1)\left(K^{4}-1\right)$ and $f: M \rightarrow N$ is a harmonic $K$-quasiconformal mapping then (i) if $n=m$ the mapping is volume decreasing, (ii) if $n \leqslant m$ the mapping is distance decreasing (up to a constant).

COROLlary 1. Let $M$ be a 2 m-dimensional complete quasi-Kaehlerian manifold with Ricci curvature bounded below and let $N$ be a $2 n$-dimensional quasi-Kaehlerian manifold with curvature conditions as in the theorem.

If $f: M \rightarrow N$ is an almost complex $K$-quasiconformal mapping then for $m=n$ the mapping is volume decreasing, for $n \leqslant m$ the mapping is distance decreasing.

If in [10] we have $B=0$, we get $\left\|f_{*}\right\|^{2} \equiv 0$, which proves the following Picard type theorem:

COROLlARY 2. Let $M$ be an m-dimensional complete Riemannian manifold with nonnegative Ricci curvature and let $N$ be as in the theorem. Then if $f: M \rightarrow N$ is a $K$-quasiconformal harmonic mapping it is reduced to a constant.

In the following example we can see that the condition that the scalar curvature must be bounded away from zero cannot be dropped.

ExAmple 1. Let $M=C$ with the flat metric and $N=C$ with the conformal metric $d s^{2}=\left(1+|z|^{2}\right) d z d \bar{z}$.

The curvature of $N$ is strictly negative everywhere, but not bounded away from zero. The identity mapping is trivially conformal but not distance decreasing.

2. The Chern-Lu formula. Let $M$ and $N$ be $C^{\infty}$ oriented Riemannian manifolds of dimension $m$ and $n$ respectively, and let $f: M \rightarrow N$ be a harmonic mapping. If the line elements are given by $d s_{M}^{2}=\sum_{i=1}^{m} \omega_{i}^{2}$ and $d s_{N}^{2}=\sum_{a=1}^{n} \omega_{a}^{* 2}$ for $M$ and $N$ respectively, and we set $\left\|f_{*}\right\|=f^{*} d s_{N} / d s_{M}$ and $f^{*} \omega_{a}^{*}=\sum_{i=1}^{m} A_{i}{ }^{a} \omega_{i}$, where $f^{*}$ is the pull-back mapping, the real analogue of Chern-Lu formula is given by [9].

$$
\frac{1}{2} \Delta\left\|f_{*}\right\| \geqslant \sum_{a, i, j} R_{i j} A_{i}^{a} A_{j}^{a}-\sum_{\substack{a, b, c, d \\ i, j}} R_{a b c d}^{*} A_{i}^{a} A_{j}^{b} A_{i}^{c} A_{j}^{d}
$$

where $R_{i j}$ are the components of the Ricci tensor for $M, R_{a b c d}^{*}$ are the components of curvature tensor of $N$ and $\Delta\left\|f_{*}\right\|$ denotes the Laplacian of $\left\|f_{*}\right\|$.

3. Mappings of bounded dilatation. The notation being as in the previous section, let $f_{*}: T_{x}(M) \rightarrow T_{f(x)}(N)$ be the tangent mapping and $h$ a metric in $N$. The tensor $f^{*} h$ is a symmetric semidefinite quadratic form on $T_{x}(M)$. With a suitable choice of 
frames in $T_{x}(M)$ and $T_{f(x)}(N)$, this quadratic form becomes

$$
f^{*} h=\sum_{i=1}^{k} \gamma_{i}^{2} \omega_{i} \circ \omega_{i} \text { at } x,
$$

where $k$ is the rank of $f_{*}$ and the positive numbers $\gamma_{i}^{2}, i=1,2, \ldots, k$, are the eigenvalues of ${ }^{t} f_{*} f_{*}$. The ratio $1_{i}=\gamma_{i} / \gamma_{i+1}$ is called the $i$ th dilatation of $f_{*}(x)$. If the first dilatation is bounded over $M$ the mapping is called of bounded dilatation [2]. The mapping is called $K$-quasiconformal if it is of maximal rank and $1_{k-1}$ is bounded over $M$ by a constant [5].

If $f_{*}\left(e_{i}\right)=A_{i}$, as in the previous section, the mapping $f_{*}: \Lambda^{p} T_{x}(M) \rightarrow \Lambda^{p} T_{f(x)}(N)$ is defined by

$$
f_{*}\left(e_{i_{1}} \wedge \cdots \wedge e_{i_{p}}\right)=f_{*}\left(e_{i_{1}}\right) \wedge \cdots \wedge f_{*}\left(e_{i_{p}}\right)
$$

and with the induced metrics in $\Lambda^{p}$ we have

$$
\left\|\Lambda^{p} f_{*}\right\|^{2}=\operatorname{trace} \Lambda^{p}\left({ }^{t} f_{*} f_{*}\right)=\sum_{1<i_{1}<\cdots<i_{p}<m} \gamma_{i_{1}}^{2} \cdots \gamma_{i_{p}}^{2}
$$

where ${ }^{t} f_{*}$ is the transpose of $f_{*}$.

In particular, $\left\|\Lambda^{2} f_{*}\right\|^{2}=\Sigma_{i<j}\left\|A_{i} \wedge A_{j}\right\|^{2}$.

For a $K$-quasiconformal mapping we have the following inequalities [4].

$$
\left[\frac{\left\|\Lambda^{q} f_{*}\right\|^{2}}{\left(\begin{array}{l}
k \\
q
\end{array}\right)}\right]^{1 / q} \leqslant\left[\frac{\left\|\Lambda^{p} f_{*}\right\|^{2}}{\left(\begin{array}{l}
k \\
p
\end{array}\right)}\right]^{1 / p} \leqslant K^{2}\left[\frac{\left\|\Lambda^{q} f_{*}\right\|^{2}}{\left(\begin{array}{l}
k \\
q
\end{array}\right)}\right]^{1 / q}
$$

for $1 \leqslant p \leqslant q \leqslant k$.

The following inequality, which follows directly from the definition of a $K$-quasiconformal mapping, will be used in the next section

$$
\left\|f_{*}\right\|^{2}<n K^{2}\left\|A_{i}\right\| \cdot\left\|A_{j}\right\|
$$

for any $i, j$ for which $\left\|A_{i}\right\|,\left\|A_{j}\right\| \neq 0$.

4. Proof of the main theorem. For the proof of Theorem 1 we need the following proposition due to S. T. Yau [10].

Proposition 3. Let $u$ be $a C^{2}$ function defined and bounded from below. Then there exists a sequence of points $\left\{q_{n}\right\} M$ such that

$$
\lim _{n \rightarrow \infty} u\left(q_{n}\right)=\operatorname{Sup} u, \quad \lim _{n \rightarrow \infty}\left\|d u\left(q_{n}\right)\right\|=0, \quad \lim _{n \rightarrow \infty} \operatorname{Sup} \Delta u\left(q_{n}\right)<0 .
$$

With the notation as in the last section, let $u=\left\|f_{*}\right\|^{2}=\Sigma_{a, i}\left(A_{i}^{a}\right)^{2}$.

The function $F=-1 / \sqrt{u+1}$ is bounded on $M$. We observe that when $F$ tends towards its supremum then $u$ also tends toward its sup. Straight computation shows that the Laplacians of $u$ and $F$ are related by

$$
\frac{1}{6}(F)^{-2} \Delta u=\frac{1}{3}(F)^{-1 / 2} \Delta F+\|d f\|^{2} .
$$


If we assume that $M$ is as in Proposition 3 then this proposition is applicable on $F$ and we have

LEMMA 1. Let $M$ be a complete Riemannian manifold with Ricci curvature bounded from below and let $u$ be a nonnegative $C^{2}$ function on $M$. There is a sequence of points $\left\{q_{n}\right\} M$ such that

$$
\lim _{n \rightarrow \infty} \operatorname{Sup}\left[u\left(q_{n}\right)+1\right]^{-2} \Delta u\left(q_{n}\right)<0, \quad \lim _{n \rightarrow \infty} u\left(q_{n}\right)=\operatorname{Sup} u .
$$

Proof of THE THEOREM. Let $f: M \rightarrow N$ be as in the theorem. At a point $x$ where $f_{*} \neq 0$, a frame $\left\{e_{1}, e_{2}, \ldots, e_{m}\right\}$ of $M$ can be chosen so that $A_{i} \perp A_{j}$, for $i \neq j$, where $A_{i}=f_{*} e_{i}$ and $i=1,2, \ldots, n$. With such an arrangement, the sectional curvature of the section spanned by $A_{i}, A_{j}$ is given by

$$
H_{i j}=\frac{\sum R_{a b c d}^{*} A_{i}^{a} A_{j}^{b} A_{i}^{c} A_{j}^{d}}{\left\|A_{i}\right\|^{2}\left\|A_{j}\right\|^{2}}
$$

so, we have

$$
\sum R_{a b c d}^{*} A_{i}^{a} A_{j}^{b} A_{i}^{c} A_{j}^{d}=\sum H_{i j}\left\|A_{i}\right\|^{2}\left\|A_{j}\right\|^{2} .
$$

If $H_{i j}^{\prime}$ and $H_{i j}^{\prime \prime}$ are defined by

$$
H_{i j}^{\prime}=\frac{H_{i j}+\left|H_{i j}\right|}{2}, \quad H_{i j}^{\prime \prime}=\frac{H_{i j}-\left|H_{i j}\right|}{2}
$$

and $\left\|A_{1}\right\|,\left\|A_{n}\right\|$ are the max and min respectively of $\left\|A_{i}\right\|$ for $i=1,2, \ldots, n$ (this can be assumed without loss of generality), we obtain from (4)

$$
\begin{aligned}
\frac{1}{2} \sum R_{a b c d}^{*} A_{i}^{a} A_{j}^{b} A_{i}^{c} A_{j}^{d} & \leqslant\left(\sum H_{i j}^{\prime}\right)\left\|A_{1}\right\|^{4}-\left(\sum\left|H_{i j}^{\prime \prime}\right|\right)\left\|A_{n}\right\|^{4} \\
& =\left[\left(\sum H_{i j}^{\prime}\right) \frac{\left\|A_{1}\right\|^{4}}{\left\|A_{n}\right\|^{4}}-\sum\left|H_{i j}^{\prime \prime}\right|\right]\left\|A_{n}\right\|^{4} .
\end{aligned}
$$

Assuming that the mapping is $K$-quasiconformal (i.e. $\left\|A_{i}\right\| /\left\|A_{n}\right\|<K$ ) that $H_{i j}^{\prime}<H$ and that $\Sigma H_{i j}^{\prime}-\Sigma\left|H_{i j}^{\prime \prime}\right|<-C$, we obtain from (5)

$$
\sum R_{a b c d}^{*} A_{i}^{a} A_{j}^{b} A_{i}^{c} A_{j}^{d} \leqslant 2\left\{\left(m(m-1)\left(K^{4}-1\right)\right) H-C\right\}\left\|A_{n}\right\|^{4} .
$$

If (as it is assumed in the theorem) we take $H<C / m(m-1)\left(K^{4}-1\right)$ (6) becomes

$$
\sum R_{a b c d}^{*} A_{i}^{a} A_{j}^{b} A_{i}^{c} A_{j}^{d} \leqslant-2 A\left\|A_{n}\right\|^{4} \text { where } A=C-m(m-1)\left(K^{4}-1\right) .
$$

Finally, from (3) we obtain

$$
\sum R_{a b c d}^{*} A_{i}^{a} A_{j}^{b} A_{i}^{c} A_{j}^{d} \leqslant-2 A\left\|f_{*}\right\|^{4} / n^{2} K^{4} .
$$

Now, we take into account the assumption that the Ricci curvatures of $M$ are bounded below by a constant $-B$. Hence

$$
\sum R_{i j} A_{i}^{a} A_{j}^{a} \geqslant-B\left\|f_{*}\right\|^{2} .
$$


Reminding, finally, that $f$ is harmonic, we have from (5), (7) and (8)

$$
\frac{1}{2} \Delta\left\|f_{*}^{2}\right\|^{2} \geqslant-B\left\|f_{*}\right\|^{2}+A\left\|f_{*}\right\|^{4} / n^{2} K^{4} \text {. }
$$

Applying Lemma 1, there is a sequence of points $\left\{q_{n}\right\} M$ such that

$$
\begin{gathered}
\lim _{n \rightarrow \infty} \sup \frac{\left\|f_{*}\right\|^{2}\left(q_{n}\right)\left[-B+\left(A / n^{2} K^{4}\right)\left\|f_{*}\right\|^{2}\left(q_{n}\right)\right]}{\left[\left\|f_{*}\right\|^{2}\left(q_{n}\right)+1\right]^{2}}<0, \\
\lim _{n \rightarrow \infty}\left\|f_{*}\right\|^{2}\left(q_{n}\right)=\sup \left\|f_{*}\right\|^{2}
\end{gathered}
$$

from which we obtain

$$
\sup \left\|f_{*}\right\|^{2} \leqslant(B / A) n^{2} K^{4}
$$

This inequality proves the theorem for the distance decreasing property. As far as the volume decreasing property is concerned comparing (2) and (9) we obtain

$$
\left[\left\|\Lambda^{n} f_{*}\right\|^{2}\right]^{1 / n} \leqslant\left|(B / A) n^{2} K^{4}\right|^{1 / n}
$$

which completes the proof of the theorem.

5. The Hermitian case. Let $M$ be a $2 m$-dimensional almost complex manifold with complex structure $J$ and Riemannian metric $g$. If the metric $q$ is invariant by $J$, i.e. $g(J X, J Y)=g(X, Y)$ for any vector fields $X$ and $Y$ the metric is called Hermitian and the manifold is called almost Hermitian. A linear connection on $M$ such that the metric tensor and the complex structure are parallel is called Hermitian connection with pure torsion tensor $T$, i.e. $T(J X, X)=T(X, J Y)$ for all vector fields $X$ and $Y$ on $M$, is called canonical connection. The existence and uniqueness of the canonical connection is assured by the general theory [1]. Let $\Phi$ be the Kaehler form of $M$, i.e. $\Phi(X, Y)=g(X, J Y)$ for all vector fields $X$ and $Y$. If the Kaehler form $\Phi$ is closed $M$ is called almost Kaehlerian manifold and if the part of $d \Phi$ of bidegree $(1,2)$ vanishes $M$ is called quasi-Kaehlerian.

It is well known that a holomorphic mapping of Kaehlerian manifolds is harmonic. This has been extended, by Lichnerowicz [8], to almost complex mappings of quasi-Kaehlerian to quasi-Kaehlerian manifolds. Corollary 1 is an immediate consequence of this proposition and the theorem.

\section{REFERENCES}

1. S. S. Chern, Characteristic classes of Hermitian manifolds, Ann. of Math. (2) 47 (1946), 85-121.

2. S. S. Chern and S. I. Goldberg, On the volume decreasing property of a class of real harmonic mappings, Amer. J. Math. 97 (1975), 133-147.

3. J. Eells, Jr. and G. H. Sampson, Harmonic mappings of Riemannian manifolds, Amer. J. Math. 86 (1964), 109-160.

4. S. I. Goldberg, T. Ishihara and N. C. Petridis, Mappings of bounded dilatation of Riemannian manifolds, J. Differential Geom. 10 (1975), 619-630.

5. S. I. Goldberg and T. Ishihara, Harmonic quasiconformal mappings of Riemannian manifolds, Bull. Amer. Math. Soc. 80 (1974), 562-566. 
6. S. I. Goldberg and Zvi Har'El, A general Schwarz lemma for Riemannian manifolds (to appear).

7. S. Kobayashi, Hyperbolic manifolds and holomorphic mappings, Dekker, New York, 1970.

8. A. Lichnerowicz, Applications harmoniques et variétés Kählériennes, Symposia Mathematica, vol. 3, Academic Press, London, 1970, pp. 341-402.

9. N. C. Petridis, A generalization of the little theorem of Picard, Proc. Amer. Math. Soc. 61 (1976), 265-271.

10. S. T. Yau, A general Schwarz lemma for Kähler manifolds, Amer. J. Math. 100 (1978), 197-203.

Department of Mathematics, Eastern Illinois Universtry, Charleston, Illinois 61920

Current address: Department of Mathematics, University of Crete, Iraklion, Crete, Greece 